\title{
System Modeling Stabilization for Mobile Display
}

\author{
Jyh-Da Wei ${ }^{*}$ and Hsu-Fu Hsiao \\ Department of Computer Science and Information Engineering, \\ College of Engineering, Chang Gung University \\ 259 Wenhwa 1st Rd., Kweishan, Taoyuan 333, Taiwan \\ *Corresponding Author: jdwei@ mail.cgu.edu.tw
}

\begin{abstract}
Mobile computing devices facilitate our daily life; however, mobile display also results in a heavy burden on our vision, and even injures our eyes. Mobile devices cause visual discomfort due to not only the screen glares but also the hand-held shake and vibration. We therefore aim to develop a stabilization technique for mobile display. This technique is expected to provide users with a comfortable interaction experience and reduce the hurt caused by screen vibration. The method we proposed is built upon system modeling. It analyzes the sensor data of acceleration and then predicts the displacement of the hand-held device. The mobile device can therefore adjust the position of the display area to compensate for vibrations. Experimental verification based on users' feedback reveals that our method successfully reduces the sight discomfort in moving environments.
\end{abstract}

Keywords: Mobile Device, Mobile Display, Stabilization, System Modeling

\section{Introduction}

Following the reports of International Data Center (IDC, http://www.idc.com), the worldwide shipments of smart phones and tablet computers will reach 1.3 billion and 240 million units, respectively, in 2015. The market of mobile computing devices, including smart phones and tablet computers, continuously increased in recent years. This situation reveals that using mobile computing devices could have engaged much time of our everyday life. Many researchers notice that mobile devices could cause ill effects to the addicts (also known as the phubbing). As indicated by Connolly et. al. [2,3], overuse of mobile devices have produced various types of social and health
Table 1. Percentages of Taiwan Students Suffering the Vision Decline.

\begin{tabular}{c|ccc}
\hline Year & Primary School & Junior High School & Senior High School \\
\hline \hline 2007 & 44.89 & 69.11 & 84.9 \\
2008 & 46.70 & 70.76 & 85.7 \\
2009 & 47.86 & 71.58 & 86.1 \\
2010 & 49.1 & 73.52 & 86.4 \\
2011 & 50.01 & 74.25 & 86.5 \\
2012 & 49.36 & 73.71 & 86.3 \\
2013 & 48.11 & 73.51 & 86.6 \\
2014 & 47.06 & 73.32 & 86.8 \\
\hline
\end{tabular}

problems. Among these problems, the most frequently we may have ever encountered are motion sickness [12] and vision damage [14] caused by hand-held shake in moving environments. Table 1 lists the percentages of Taiwan students who suffer the vision decline, i.e., with at least one of the eyes not reaching 20/20 at eye exams. According to these data released by the ministry of Education, Taiwan (http://www.edu.tw), a very high rate of school children and teenagers have to correct their vision. Serious sight problem like this can also put the blame on the overuse of mobile computing devices [15].

To release the discomfort of eyes and reduce the risk of vision damage, we propose a method to stabilize the display of a mobile device in this paper. Fig 01 illustrates our solution. We set an active area on the screen for displaying the media content, e.g., pictures, text segments, web pages and the output of other apps. Outside the active area is a buffer zone for this display area to move to compensate for the vibrations of screen. We provide three parameters for this stabilization mechanism, i.e., the moving range "d", the scale factor "c", and the reset time " $\mathrm{t}$ ". Parameter $d$ indicates the buffer distance from the edge of the screen to the active area for display. If $\mathrm{d}$ is assigned zero, the active zone will move without buffers. Parameter $c$ is a scale factor, which allows the users to adjust the scale for each 


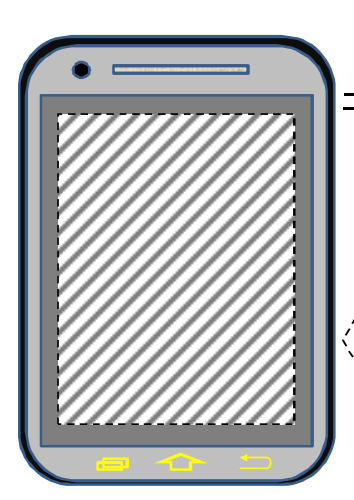

\section{Parameters - d: moving range $(\mathrm{cm})$ t: reset time (sec)}

d
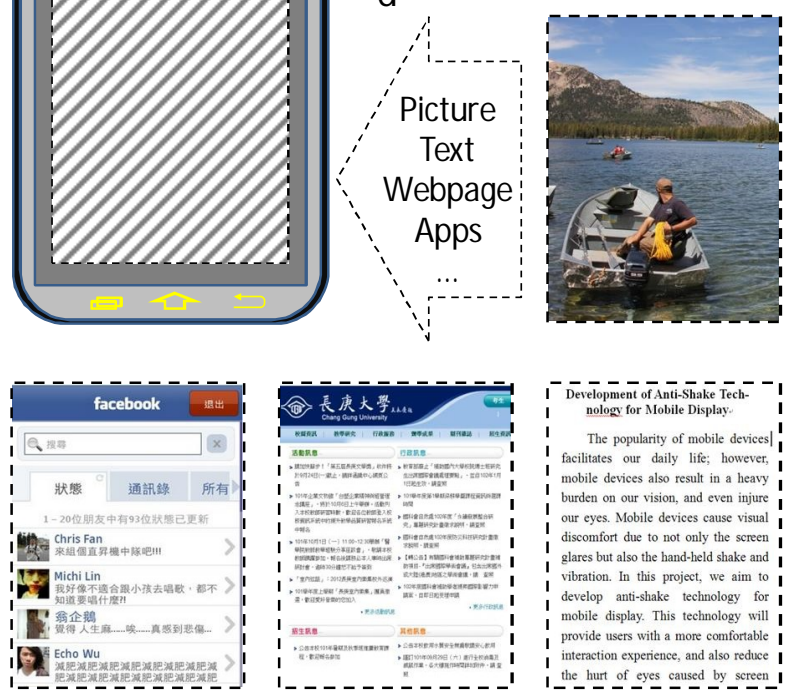

Fig. 1. Stabilization Method for Mobile Device Display.

compensation move. Finally, parameter $t$ introduces a period not to run the compensation process. The timer of this period will be reset and the active area will return to the center of the screen whenever the screen is touched or the device is shaken with a displacement more than a threshold. This way the user can interact with the mobile device as usual.

We take the AR (auto-regressive) model for system modeling [8] and use the recurrent algorithm of least-squares estimation (LSE) [5] to calculate the coefficients of the model. The model coefficients are updated in real time and we can therefore predict the displacement at each moment. We applied this stabilization technique on displaying a picture, a text segment, and a video for users to test. The positive feedback from the users successively verified the feasibility of this stabilization technique.

\section{Related Work}

Stabilization of media access is a valuable research topic. Practical techniques have been developed for image and video capture. We can also find some related research to improve the quality of mobile text display.

\subsection{Stabilization Techniques for Image Capture}

Hand-held vibration is a serious problem for digital cameras. Stabilization techniques for image and video recording are thus developed by famous electric companies

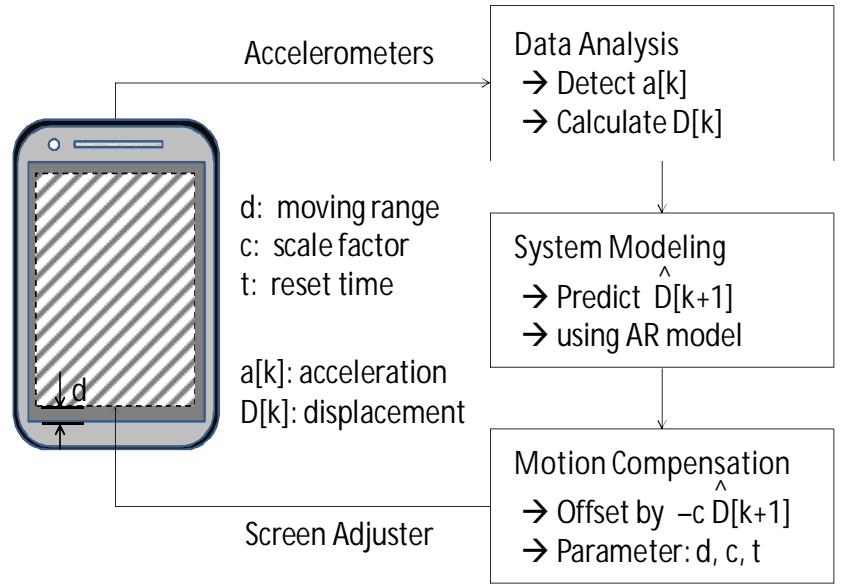

Fig. 2. System Infrastructure of the Stabilization Mechanism

[10,11]. The image stabilization (IS) technique of Canon and the vibration reduction (VR) technique of Nikon are examples that use damping systems on the camera lens to enhance the image quality during capture. Similar method was also developed by Sony and Monica; nevertheless, they applied the damping mechanism on the CCD unit. Several offline processing algorithms were also proposed to reduce the vibration of video $[4,13]$. These algorithms provide software solution that aligns and clips subareas in conjoint frames and thus regenerate a stable video.

Although these techniques perform well for image and video capture, they are not available for mobile device display because neither can we attach a damping screen on the devices nor is an offline post-process allowed during user operation. Therefore, the only way to solve hand-held shake is to predict the displacement of moving and then compensate it on the opposite direct.

\subsection{Stabilization Techniques for Mobile Text Display}

Early in 2007, Barnard et. al. had noticed that handheld vibrations could interference text reading in moving environments [1]. The corresponding researches to facilitate text reading on mobile devices include enlarging the text size dynamically [7] and magnifying local area of the content [9]. Analysis of walk types [6] is an advanced research that increases the performance of these solutions.

In this paper, we propose a system modeling based stabilization technique. This method predicts and offsets the next move of the shake and thus stabilizes the display area in the center of the screen. Aside from text display, our method suits for displaying all other kinds of media content. 


\section{System Modeling Stabilization Technique}

The proposed stabilization technique consists of three pipelined units. As Fig. 2 shows, these three units take charge of motion analysis, motion prediction and motion compensation respectively. Here we introduce how these units work and then state the experiments we conduct to validate our system.

\subsection{Auto-Regression System Model}

The first functional unit analyzes accelerometer data to estimate the displacement of the mobile device. The acceleration is divided into $\mathrm{X}$ and $\mathrm{Y}$ components and then adjusted by the gyroscope to remove the gravity effects. The stabilization mechanism processes $\mathrm{X}$ and $\mathrm{Y}$ data separately. The estimation equation that both applies to $\mathrm{X}$ and $\mathrm{Y}$ axes is given by eqn (1) with system parameters $\rho$ and $\gamma$, where $a$ and $D$ sequences stands for the acceleration and the displacement, respectively.

$$
\begin{aligned}
& D[k] \\
& =\rho \cdot t \cdot v[k] \\
& \approx \rho \cdot t(v[k-1]+t \cdot a[k]) \\
& =\rho \cdot D[k-1]+\gamma \cdot a[k] .
\end{aligned}
$$

The second functional unit then uses system modeling methods to predict the next displacement value. An auto-regression model with weight set $\theta$ 's is established as follows, where system parameter $n$ indicates the window size of filtering.

$$
\widehat{D}[k+1]=\sum_{i=0}^{n-1} \theta_{i} \cdot D[k-i]
$$

Motion prediction, at time $k$, can be realized by eqn (2) and the following recurrent LSE equations (eqn (3) (5)):

$$
\begin{aligned}
& \phi_{k}=\left[\begin{array}{llll}
D[k-1] & D[k-2] & \cdots & D[k-n
\end{array}\right]^{T} \\
& P_{k}=P_{k-1}-\frac{P_{k-1} \phi_{k} \phi_{k}^{T} P_{k-1}}{1+\phi_{k}{ }^{T} P_{k-1} \phi_{k}} \\
& \hat{\theta}_{k}=\hat{\theta}_{k-1}+P_{k} \phi_{k}\left(y_{k}-\phi_{k}^{T} \hat{\theta}_{k-1}\right)
\end{aligned}
$$

Finally, we use the third unit to adjust the position of the active display area. As stated above in Section 1, this module works following three parameters, i.e., the moving range "d", the scale factor "c" and the reset time " $t$ ". The compensatory offset $C[k+1]$ can be calculated by eqn (6).

$$
C[k+1]=-c \cdot \widehat{D}[k+1]
$$

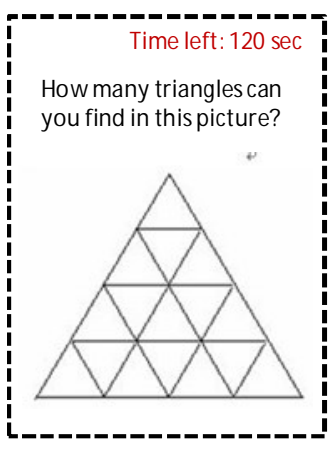

(a)

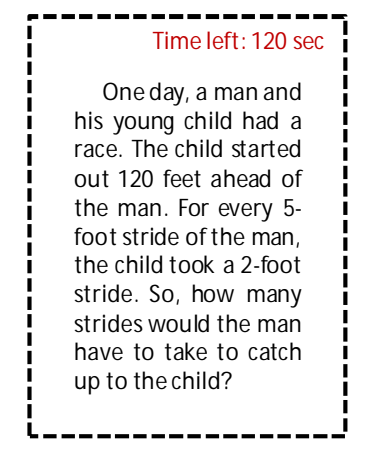

(b)

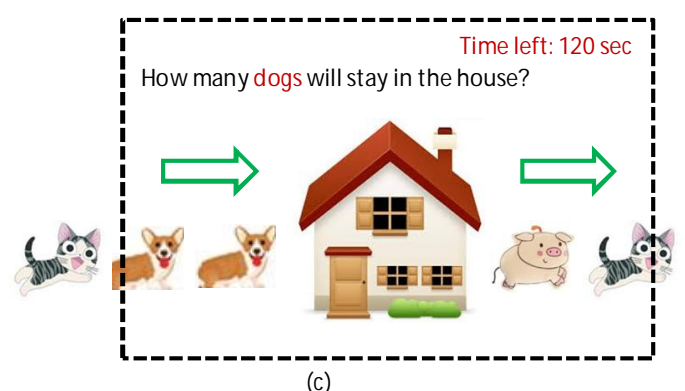

(c)
Fig. 3. Three Types of Questions for Eye Comfort Tests.

\subsection{User Test}

We design a practice operation to verify the proposed stabilization technique. As Fig. 3 shows, three types of questions involving (a) a picture-type graphic problem, (b) a text-type math problem, and (c) a video-type counting problem are exhibited to the users. Each of these problems takes two minutes for the users to answer. The correctness of the answers is not important in our tests, but we record the user's feedback after the tests. Users have to reply how comfortable they feel about the display during the test. The scores can be from 1 to 5 - a higher number implies a better experience. We then compare the scores resulting from the cases when the stabilization mechanism is turned on and off. The moving environments include walking, taking bus, taking Taipei MRT, and taking MRT with a side-sitting posture.

\section{Experimental Resluts}

We conducted the experiments using an hTC Nexus 9 tablet computer for it is with the standard Android 6.0 operation system. The userbase includes 20 college students with equal rates of girls and boys. Table 2 lists the experimental results. According to the final record, our stabilization technique provided more comfortable feeling in all moving modes. We can also find that side-sitting posture is really not suitable to use a mobile device. 
Table 2. Eye Comfort Scores Collected from Users' Feedback.

\begin{tabular}{c|cc|cc|cc}
\hline & \multicolumn{2}{|c|}{ Picture } & \multicolumn{2}{|c|}{ Text } & \multicolumn{2}{c}{ Video } \\
\hline ModelStabilization & on & off & on & off & on & off \\
\hline Walking & 4.2 & 3.9 & 4.4 & 3.8 & 4.1 & 3.2 \\
Bus & 4.4 & 4.1 & 4.6 & 4.4 & 4.5 & 4.2 \\
MRT & 4.4 & 4.0 & 4.5 & 4.3 & 4.4 & 4.1 \\
MRT (side-sitting) & 3.9 & 3.3 & 4.1 & 3.1 & 3.7 & 3.2 \\
Average & 4.23 & 3.83 & 4.40 & 3.90 & 4.18 & 3.68 \\
\hline
\end{tabular}

\section{Conclusions}

In this paper, we propose a system modeling based stabilization technique for mobile display. Our method consists of three pipelined units, i.e., the motion analysis, the motion prediction and the motion compensation units. The first unit analyzes accelerometer data to estimate the displacement of the mobile device. The second unit then uses system modeling methods to predict the next displacement of moving. Finally, the third unit adjusts the position of the display area to compensate for vibrations. Experiments were conducted and the users' feedback was collected. Experimental results verify that this stabilization technique for mobile display can make our eyes feel more comfortable while using mobile computing devices.

\section{Acknowledgment}

This work was supported in part by the Ministry of Science and Technology under the Grant MOST 104-2221E-182-063.

\section{References}

(1) L. Barnard, J. S. Yi, J. A. Jacko, and A. Sears. Capturing the effects of context on human performance in mobile computing systems. Personal and Ubiquitous Computing, 11(2):81-96, Jan. 2007.

(2) R. W. Connolly. Beyond good and evil impacts: Rethinking the social issues components in our computing curricula. In Proc. the 16th Annual Joint Conference on Innovation and Technology in Computer Science Education, ITiCSE '11, pages 228-232, 2011.

(3) R. W. Connolly. Criticizing and modernizing computing curriculum: The case of the web and the social issues courses. In Proc. the Seventeenth Western Canadian Conference on Computing Education, WCCCE'12, pages 52-56, 2012.
(4) R. Fergus, B. Singh, A. Hertzmann, S. T. Roweis, and W. T. Freeman. Removing camera shake from a single photograph. ACM Transactions on Graphics, 25(3):787-794, July 2006.

(5) L. Fu and P. Li. The research survey of system identification method. In Proc. 5th International Conference on Intelligent Human-Machine Systems and Cybernetics (IHMSC), pages 397-401, 201

(6) M. Goel, L. Findlater, and J. Wobbrock. Walktype: Using accelerometer data to accomodate situational impairments in mobile touch screen text entry. In Proc. SIGCHI Conference on Human Factors in Computing Systems, pages 2687-2696, May 2012.

(7) S. K. Kane, J. O. Wobbrock, and I. E. Smith. Getting off the treadmill: Evaluating walking user interfaces for mobile devices in public spaces. In Proc. 10th International Conference on Human Computer Interaction with Mobile Devices and Services, pages 109-118, Sept. 2008.

(8) C. A. Kluever. Dynamic Systems: Modeling, Simulation, and Control. WILEY, 1st edition, 2015.

(9) Z. Li, S. Pundlik, and G. Luo. Stabilization of magnified videos on mobile device for visually impaired. In Proc. Computer Vision and Pattern Recognition Workshops, pages 54-55, June 2013.

(10) S. Liu, L. Yuan, P. Tan, and J. Sun. Bundled camera paths for video stabilization. ACM Transactions on Graphics, 32(4):78:1-78:10, July 2013.

(11) N. Mansurov. Lens stabilization vs in-camera stabilization. Photographylife: articles, news, reviews, Feb. 2012.

(12) T. A. Stoffregen, Y.-C. Chen, and F. C. Koslucher. Motion control, motion sickness, and the postural dynamics of mobile devices. Experimental Brain Research, 232:1389-1397, Apr. 2014.

(13) C. Tang, X. Yang, L. Chen, and G. Zhai. A fast video stabilization algorithm based on block matching and edge completion. In Proc. IEEE 13th International Workshop on Multimedia Signal Processing (MMSP), pages 1-5, 2011.

(14) M. Vinnikov, R. S. Allison, and D. Swierad. Real-time simulation of visual defects with gaze-contingent display. In Proc. the 2008 Symposium on Eye Tracking Research \& Applications (ETRA), pages 127-130, 2008.

(15) R. S. Wagner. Smartphones, video display terminals, and dry eye disease in children. Journal of pediatric ophthalmology and strabismus, 51:76, Mar. 2014. 